\title{
Influence of probiotics, prebiotics, synbiotics and bioactive phytochemicals on the formulation of functional yogurt
}

\begin{abstract}
The new concept of functional foods has led to the varieties in the production of foods that deliver not only basic nutrition, but can also warrant good health and longevity. Yogurt has become one of the prevalent choices and considered as a healthy food since it provides excellent sources of essential nutrients. As the popularity of yogurt continues to grow, manufacturers and scientists continuously investigate the value adding ingredients such as probiotics, prebiotics and different kinds of plant extracts to produce functional yogurt comprising extra beneficial properties than the conventional yogurt. This review summarises the current knowledge on functional yogurt, applications and roles of probiotic, prebiotic and synbiotic in yogurt as well as the effects of phytochemicals added in innovative yogurt products. Their important properties were focused based on significance influences on quality and sensory attributes of yogurt products and associated health aspects.
\end{abstract}

Keyword: Yogurt; Functional food; Probiotic; Prebiotic; Synbiotic; Phytochemical 